\title{
Ghosts of Company
}

The young barmaid held her gaze,

When he raised his mask,

To salute with mercurial gusto,

Heroes of the past.

Now he is laid down to rest,

Resolute and steadfast.

Amidst "the fearless",

His dearest left cursing his craft.

At dusk, fading from actuality,

Not the first,

Not the last,

All just ghosts of company,

Hiding themselves behind a glass.

Before an altar,

We must not falter,

For 'tis He himself who laughs last.

Time. Gentlemen. Please!

My, oh my...

How it has passed.

\section{Owen P O'Sullivan}

Correspondence to Owen P O'Sullivan, National Forensic Mental Health Service, Central Mental Hospital, Dundrum, Dublin 14, Ireland; owenosullivan@rcsi.ie

Competing interests None.

Provenance and peer review Not commissioned; internally peer reviewed.

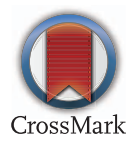

To cite O'Sullivan OP. Med Humanit 2017;43:e10.

Published Online First 20 October 2016

Med Humanit 2017:43:e10. doi:10.1136/medhum-2016-011103 